\title{
Density functional energy decomposition into one- and two-atom contributions
}

\author{
Sergei F. Vyboishchikov, ${ }^{\text {a) }}$ Pedro Salvador, and Miquel Duran \\ Institut de Química Computacional, Campus de Montilivi, Universitat de Girona, 17071 Girona, Catalonia, \\ Spain
}

(Received 7 March 2005; accepted 25 April 2005; published online 6 July 2005)

\begin{abstract}
The present work provides a generalization of Mayer's energy decomposition for the density-functional theory (DFT) case. It is shown that one- and two-atom Hartree-Fock energy components in Mayer's approach can be represented as an action of a one-atom potential $V^{A}$ on a one-atom density $\rho^{A}$ or $\rho^{B}$. To treat the exchange-correlation term in the DFT energy expression in a similar way, the exchange-correlation energy density per electron is expanded into a linear combination of basis functions. Calculations carried out for a number of density functionals demonstrate that the DFT and Hartree-Fock two-atom energies agree to a reasonable extent with each other. The two-atom energies for strong covalent bonds are within the range of typical bond dissociation energies and are therefore a convenient computational tool for assessment of individual bond strength in polyatomic molecules. For nonspecific nonbonding interactions, the two-atom energies are low. They can be either repulsive or slightly attractive, but the DFT results more frequently yield small attractive values compared to the Hartree-Fock case. The hydrogen bond in the water dimer is calculated to be between the strong covalent and nonbonding interactions on the energy scale. (C) 2005 American Institute of Physics. [DOI: 10.1063/1.1935511]
\end{abstract}

\section{INTRODUCTION}

The energy or the strength of the chemical bond is a key notion in quantum chemistry. In most cases, the bond dissociation energy is used as a measure for the bond strength, since it is a well-defined observable and can be both measured experimentally and calculated using quantum-chemical techniques. This is usually done using the supermolecular approach by evaluating the energy difference $E(A B)-E(A)$ $-E(B)$. This approach requires a clear separability of the molecule $A B$ into two isolated fragments $A$ and $B$. This separability is often possible, but not applicable for the bonds that form a part of a ring system because the fragments are not well defined then. For the same reason, the dissociation energy is not applicable for weak intramolecular interactions, such as agostic and interligand interactions in organometallic complexes or intramolecular hydrogen bonds. The question about the existence and the strength of such an intramolecular interaction can be addressed by other means. For example, Bader's atoms-in-molecules theory ${ }^{1}$ postulates the presence of a chemical bond between two atoms when they are connected by a path of the gradient vector field $\nabla \rho$. Similarly, a bond is found within the natural bond orbital (NBO) analysis, ${ }^{2}$ when a corresponding two-center bond orbital is detected. Both approaches turned out to be extremely useful, but they do not yield a direct energetic measure of bond strength and usually fail to deliver any meaningful information for weak interactions, when no bond path or no NBO can be located.

In this connection, for interpretation of the molecular

\footnotetext{
a) Author to whom correspondence should be addressed. Fax: +3497241 8356. Electronic mail: vybo@iqc.udg.es
}

structure it would be very useful to obtain an energetic measure of a bond strength using the approaches based on the decomposition of the total energy of a molecule into a sum of one-, two-, and possibly many-atom contributions. Once popular in the past for semiempirical methods, ${ }^{3}$ such decomposition is no longer trivial for ab initio methods due to the presence of four-center two-electron integrals in the totalenergy expression.

Recently, Mayer proposed an energy decomposition approach ["chemical energy component analysis" (CECA)], which allows dividing the total Hartree-Fock energy into one- and two-atom contributions, $E^{\mathrm{HF}} \approx \Sigma_{A} E(A)$ $+\sum_{A<B} E(A B)$. The approximate, but rather accurate, CECA decomposition makes use of the one-atom $\hat{P}^{A}=\Sigma_{\mu \nu \epsilon A}|\mu\rangle\left(S^{-1}\right)_{\mu \nu}^{A}\langle\nu| \quad$ and two-atom $\hat{P}^{A B}$ $=\sum_{\mu \nu \in A B}|\mu\rangle\left(S^{-1}\right)_{\mu \nu}^{A B}\langle\nu|$ projection operators, where $\left(S^{-1}\right)^{A}$ and $\left(S^{-1}\right)^{A B}$ are the inverse one-atom and two-atom overlap matrices, respectively, in order to condense the three- and fourcenter integrals to one- and two-center ones. Typically, bound atoms exhibit strongly negative diatomic energies, while nonbound or remote atoms have very low negative or positive contributions. Albeit useful in many respects, the two-atom energies obtained for bound atoms within Mayer's original scheme are too high in absolute value to be compared to meaningful bond energies chemists are accustomed to. This was a motivation to search for other ways of the total-energy decomposition, which culminated to another paper, ${ }^{5}$ where by assigning various terms of the total Hartree-Fock energy expression to different atoms, Mayer was able to exactly decompose it into one- and two-atom components. The two-atom energies obtained by the latter procedure are of the order of magnitude of dissociation en- 
ergies and can be directly related to bond energies within chemists' common sense.

It should be noted that in the meantime, several related, but different, decomposition schemes have been published, which are based, most notably, on the Bader partitioning into atomic basins, ${ }^{6-8}$ or on "fuzzy atoms." 8,9 In these methods the partitioning is done in the three-dimensional physical space, rather than in the Hilbert space as is the case in the CECA scheme. An early work by Sierraalta and Frenking ${ }^{10}$ belongs to the same group. On the other hand, Hamza and Mayer later extended the CECA approach by further subdividing the two-atom energy $E(A B)$ into electrostatic, exchange, overlap, and basis extension components. ${ }^{11}$

So far, all these partitioning schemes (except for the recent work ${ }^{8}$ ) have been based on the Hartree-Fock approximation. Whereas the Mayer-type partitioning is also principally possible for correlated $a b$ initio methods, the decomposition of the total energy within the densityfunctional theory (DFT) is not straightforward. On the other hand, it is highly desired to generalize the Mayer partitioning for the case of the DFT energy, since the DFT is clearly a method of choice for many highly interesting chemical systems including transition-metal complexes. Hence, in the present work we suggest a simple way of decomposing the DFT energy in the same spirit.

\section{HARTREE-FOCK ENERGY DECOMPOSITION}

First, let us consider the total Hartree-Fock energy expression. We will restrict ourselves to closed-shell systems with real wave functions, keeping in mind that the generalization to the open-shell case is trivial. The Hartree-Fock energy is given by the sum of nuclear repulsion $E_{\mathrm{NN}}$, kinetic energy $E_{\text {kin }}$, electron-nuclear attraction $E_{\mathrm{eN}}$, Coulomb (electron repulsion) $E_{C}$, and exchange $E_{x}$ components (The (11|22) convention for two-electron integrals is used throughout the paper),

$$
\begin{aligned}
& E_{\mathrm{NN}}=\sum_{A<B} \frac{Z_{A} Z_{B}}{R_{A B}}, \\
& E_{\mathrm{kin}}=\sum_{\mu \nu} D_{\mu \nu}\langle\nu|\hat{T}| \mu\rangle, \\
& E_{\mathrm{eN}}=-\sum_{A} \sum_{\mu \nu}^{\text {all }} D_{\mu \nu}\left\langle\nu\left|\frac{Z_{A}}{r_{A}}\right| \mu\right\rangle, \\
& E_{C}=\frac{1}{2} \sum_{\mu \nu}^{\text {all }} \sum_{\rho \sigma}^{\text {all }} D_{\mu \nu} D_{\rho \sigma}(\mu \nu \mid \rho \sigma), \\
& E_{x}=-\frac{1}{4} \sum_{\mu \nu}^{\text {all }} \sum_{\rho \sigma}^{\text {all }} D_{\mu \nu} D_{\rho \sigma}(\mu \rho \mid \nu \sigma),
\end{aligned}
$$

where $D_{\mu \nu}$ is an element of the total spinless charge-density bond-order matrix calculated from the molecular-orbital (MO) coefficients $C_{\mu}^{i}$ according to the conventional rule,

$$
D_{\mu \nu}=2 \sum_{i}^{\text {occ. }} C_{\mu}^{i} C_{\nu}^{i} .
$$

Instead of repeating Mayer's derivation of one- and twoatom contributions, we wish to take a different look at his results. We introduce a one-atom spinless density-matrix function $\rho^{A}\left(\mathbf{r} \mid \mathbf{r}^{\prime}\right)$ and a one-atom density $\rho^{A}(\mathbf{r})$ as follows:

$$
\begin{aligned}
& \rho^{A}\left(\mathbf{r} \mid \mathbf{r}^{\prime}\right)=\sum_{\mu \in A} \sum_{\nu}^{\text {all }} D_{\mu \nu} \chi_{\nu}(\mathbf{r}) \chi_{\mu}\left(\mathbf{r}^{\prime}\right), \\
& \rho^{A}(\mathbf{r})=\rho^{A}(\mathbf{r} \mid \mathbf{r})=\sum_{\mu \in A} \sum_{\nu}^{\text {all }} D_{\mu \nu} \chi_{\nu}(\mathbf{r}) \chi_{\mu}(\mathbf{r}),
\end{aligned}
$$

such that the total density is equal to the sum to one-atom contributions, $\rho(\mathbf{r})=\Sigma_{A} \rho^{A}(\mathbf{r})$. Further, we introduce a oneatom nuclear attraction operator $V_{N}^{A}$,

$$
V_{N}^{A}=-Z_{A} / r_{A}
$$

Then, the electron-nuclear attraction $E_{\mathrm{eN}}^{A B}$ between two atoms $A$ and $B$ will be given by the interaction of $A$-nuclear attraction operator $V_{N}^{A}$ with the $B$-atom density $\rho^{B}$, and vice versa,

$$
\begin{aligned}
E_{\mathrm{eN}}^{A B}= & \int\left[V_{N}^{A}(\mathbf{r}) \rho^{B}\left(\mathbf{r} \mid \mathbf{r}^{\prime}\right)\right]_{\mathbf{r}^{\prime}=\mathbf{r}} d \mathbf{r} \\
& +\int\left[V_{N}^{B}(\mathbf{r}) \rho^{A}\left(\mathbf{r} \mid \mathbf{r}^{\prime}\right)\right]_{\mathbf{r}^{\prime}=\mathbf{r}} d \mathbf{r}=\int V_{N}^{A}(\mathbf{r}) \rho^{B}(\mathbf{r}) d \mathbf{r} \\
& +\int V_{N}^{B}(\mathbf{r}) \rho^{A}(\mathbf{r}) d \mathbf{r} .
\end{aligned}
$$

Similarly, the one-atom contribution is $E_{\mathrm{eN}}^{A}=\int V_{N}^{A}(\mathbf{r}) \rho^{A}(\mathbf{r}) d \mathbf{r}$. The total electron-nuclear attraction is $E_{\mathrm{eN}}=\Sigma_{A} E_{\mathrm{eN}}^{A}$ $+\sum_{A<B} E_{\mathrm{eN}}^{A B}$. Inserting the above expressions for $V_{N}^{A}$ and $\rho^{A}$, we obtain

$$
\begin{aligned}
E_{\mathrm{eN}}^{A B}= & -\int \frac{Z_{A}}{r_{A}} \sum_{\mu \in B} \sum_{\nu}^{\text {all }} D_{\mu \nu} \chi_{\nu}(\mathbf{r}) \chi_{\mu}(\mathbf{r}) d \mathbf{r} \\
& -\int \frac{Z_{B}}{r_{B}} \sum_{\mu \in A} \sum_{\nu}^{\text {all }} D_{\mu \nu} \chi_{\nu}(\mathbf{r}) \chi_{\mu}(\mathbf{r}) d \mathbf{r} \\
= & -\sum_{\mu \in B} \sum_{\nu} D_{\mu \nu}\left\langle\nu\left|\frac{Z_{A}}{r_{A}}\right| \mu\right\rangle-\sum_{\mu \in A} \sum_{\nu}^{\text {all }} D_{\mu \nu}\left\langle\nu\left|\frac{Z_{B}}{r_{B}}\right| \mu\right\rangle
\end{aligned}
$$

and analogously

$$
\begin{aligned}
E_{\mathrm{eN}}^{A} & =-\int \frac{Z_{A}}{r_{A}} \sum_{\mu \in A} \sum_{\nu}^{\text {all }} D_{\mu \nu} \chi_{\nu}(\mathbf{r}) \chi_{\mu}(\mathbf{r}) d \mathbf{r} \\
& =-\sum_{\mu \in A} \sum_{\nu}^{\text {all }} D_{\mu \nu}\left\langle\nu\left|\frac{Z_{A}}{r_{A}}\right| \mu\right\rangle,
\end{aligned}
$$

which is identical to the electron-nuclear attraction terms in Eqs. (5) and (6) in Mayer's paper.

In order to decompose the Coulomb energy $E_{C}$, we introduce the one-atom Coulomb repulsion operator $J^{A}$ simply as a half of the electrostatic potential created by the one-atom density $\rho^{A}$, 


$$
J^{A}(\mathbf{r})=\frac{1}{2} \int \frac{\rho^{A}\left(\mathbf{r}^{\prime}\right)}{\left|\mathbf{r}-\mathbf{r}^{\prime}\right|} d \mathbf{r}^{\prime} .
$$

Using $J^{A}$ in a similar way to Eq. (4) one obtains the following one- and two-atom Coulomb energy expressions:

$$
\begin{aligned}
E_{C}^{A B} & =\int\left[J^{A}(\mathbf{r}) \rho^{B}\left(\mathbf{r} \mid \mathbf{r}^{\prime}\right)\right]_{\mathbf{r}^{\prime}=\mathbf{r}} d \mathbf{r}+\int\left[J^{B}(\mathbf{r}) \rho^{A}\left(\mathbf{r} \mid \mathbf{r}^{\prime}\right)\right]_{\mathbf{r}^{\prime}=\mathbf{r}} d \mathbf{r} \\
& =\int J^{A}(\mathbf{r}) \rho^{B}(\mathbf{r}) d \mathbf{r}+\int J^{B}(\mathbf{r}) \rho^{A}(\mathbf{r}) d \mathbf{r} \\
& =\iint \frac{\rho^{A}\left(\mathbf{r}^{\prime}\right) \rho^{B}(\mathbf{r})}{\left|\mathbf{r}-\mathbf{r}^{\prime}\right|} d \mathbf{r}^{\prime} d \mathbf{r} \\
& =\sum_{\mu \in A} \sum_{\rho \in B} \sum_{\nu, \sigma}^{\text {all }} D_{\mu \nu} D_{\rho \sigma}(\mu \nu \mid \rho \sigma), \\
E_{C}^{A} & =\int J^{A}(\mathbf{r}) \rho^{A}(\mathbf{r}) d \mathbf{r} \\
& =\frac{1}{2} \iint \frac{\rho^{A}\left(\mathbf{r}^{\prime}\right) \rho^{A}(\mathbf{r})}{\left|\mathbf{r}-\mathbf{r}^{\prime}\right|} d \mathbf{r}^{\prime} d \mathbf{r} \\
& =\frac{1}{2} \sum_{\mu, \rho \in A} \sum_{\nu, \sigma}^{\text {all }} D_{\mu \nu} D_{\rho \sigma}(\mu \nu \mid \rho \sigma) .
\end{aligned}
$$

The Coulomb energy obtained in such a way is also identical to the corresponding terms in Eqs. (5) and (6) in the original paper. $^{5}$

In order to introduce a one-atom exchange operator, let us first recall the well-known expression for the total Hartree-Fock exchange through the first-order density matrix,

$$
E_{x}=-\frac{1}{4} \iint \frac{\rho\left(\mathbf{r}, \mathbf{r}^{\prime}\right) \rho\left(\mathbf{r}^{\prime}, \mathbf{r}\right)}{\left|\mathbf{r}-\mathbf{r}^{\prime}\right|} d \mathbf{r} d \mathbf{r}^{\prime} .
$$

After decomposing $\rho\left(\mathbf{r} \mid \mathbf{r}^{\prime}\right)$ and $\rho\left(\mathbf{r}^{\prime} \mid \mathbf{r}\right)$ in the numerator into atomic contributions according to Eq. (2), the following partioning of the exchange energy is obtained:

$$
\begin{aligned}
E_{x}= & -\frac{1}{4} \sum_{A} \sum_{B} \iint \frac{\rho^{A}\left(\mathbf{r}, \mathbf{r}^{\prime}\right) \rho^{B}\left(\mathbf{r}^{\prime}, \mathbf{r}\right)}{\left|\mathbf{r}-\mathbf{r}^{\prime}\right|} d \mathbf{r} d \mathbf{r}^{\prime} \\
= & -\frac{1}{4} \sum_{\rho \in B} \sum_{\nu} \sum_{\mu \in A} \sum_{\sigma}^{\text {all }} D_{\mu \sigma} D_{\rho \nu}(\mu \nu \mid \rho \sigma) \\
= & -\frac{1}{4} \sum_{A} \sum_{\mu, \rho \in A} \sum_{\nu, \sigma}^{\text {all }} D_{\mu \sigma} D_{\rho \nu}(\mu \nu \mid \rho \sigma) \\
& -\frac{1}{2} \sum_{A<B} \sum_{\rho \in B} \sum_{\mu \in A} \sum_{\nu, \sigma}^{\text {all }} D_{\mu \sigma} D_{\rho \nu}(\mu \nu \mid \rho \sigma) .
\end{aligned}
$$

Then the two-atom exchange energy is given by

$$
E_{x}^{A B}=-\frac{1}{2} \sum_{\mu \in A} \sum_{\rho \in B} \sum_{\sigma, \nu}^{\text {all }} D_{\mu \sigma} D_{\rho \nu}(\mu \nu \mid \rho \sigma),
$$

which is again identical to the exchange part of the Eq. (6) in Mayer's work. ${ }^{5}$
On the other hand, the one-atom exchange operator $K^{A}$ can be defined through its action on an arbitrary one-electron function $f(\mathbf{r})$ using the one-atom density matrix as follows:

$$
\begin{aligned}
K^{A}(\mathbf{r}) f(\mathbf{r}) & =-\frac{1}{4} \int \frac{\rho^{A}\left(\mathbf{r} \mid \mathbf{r}^{\prime}\right) f\left(\mathbf{r}^{\prime}\right)}{\left|\mathbf{r}-\mathbf{r}^{\prime}\right|} d \mathbf{r}^{\prime} \\
& =-\frac{1}{4} \sum_{\mu \in A} \sum_{\sigma}^{\text {all }} D_{\mu \sigma} \int \frac{\chi_{\mu}\left(\mathbf{r}^{\prime}\right) \chi_{\sigma}(\mathbf{r}) f\left(\mathbf{r}^{\prime}\right)}{\left|\mathbf{r}-\mathbf{r}^{\prime}\right|} d \mathbf{r}^{\prime} .
\end{aligned}
$$

Using this definition, the one- and two-atom exchange components can be written in the following manner:

$$
\begin{aligned}
& E_{x}^{A B}= \int\left[K^{A}(\mathbf{r}) \rho^{B}\left(\mathbf{r} \mid \mathbf{r}^{\prime}\right)\right]_{\mathbf{r}^{\prime}=\mathbf{r}} d \mathbf{r} \\
&+\int\left[K^{B}(\mathbf{r}) \rho^{A}\left(\mathbf{r} \mid \mathbf{r}^{\prime}\right)\right]_{\mathbf{r}^{\prime}=\mathbf{r}} d \mathbf{r}, \\
& E_{x}^{A}=\int\left[K^{A}(\mathbf{r}) \rho^{A}\left(\mathbf{r} \mid \mathbf{r}^{\prime}\right)\right]_{\mathbf{r}^{\prime}=\mathbf{r}} d \mathbf{r} .
\end{aligned}
$$

Technically, the above analysis did not reveal any new results, but it demonstrates that all the potential-energy terms $E_{Y}^{A B}$ in Mayer's energy two-atom partitioning can be presented as the action of a properly chosen effective one-atom operator $\hat{Y}^{A}$ of atom $A$ on the density matrix $\rho^{B}\left(\mathbf{r} \mid \mathbf{r}^{\prime}\right)$ of atom $B$, and vice versa,

$$
\begin{aligned}
E_{Y}^{A B}= & \int\left[\hat{Y}^{A}(\mathbf{r}) \rho^{B}\left(\mathbf{r} \mid \mathbf{r}^{\prime}\right)\right]_{\mathbf{r}^{\prime}=\mathbf{r}} d \mathbf{r} \\
& +\int\left[\hat{Y}^{B}(\mathbf{r}) \rho^{A}\left(\mathbf{r} \mid \mathbf{r}^{\prime}\right)\right]_{\mathbf{r}^{\prime}=\mathbf{r}} d \mathbf{r} .
\end{aligned}
$$

With the help of the nuclear attraction operator $V_{N}^{A}$ [Eq. (3)], the Coulomb repulsion operator $J^{A}$ [Eq. (5)], and the oneatom exchange operator $K^{A}$ [Eq. (7)], Mayer's total one- and two-atom energies can be rewritten as follows:

$$
\begin{aligned}
E_{A}^{\mathrm{HF}}= & \sum_{\mu \in A} \sum_{\nu \in A} D_{\mu \nu}\langle\nu|\hat{T}| \mu\rangle \\
& +\int\left[\left(V_{N}^{A}+J^{A}+K^{A}\right) \rho^{A}\left(\mathbf{r} \mid \mathbf{r}^{\prime}\right)\right]_{\mathbf{r}^{\prime}=\mathbf{r}} d \mathbf{r}, \\
E_{A B}^{\mathrm{HF}}= & \frac{Z_{A} Z_{B}}{R_{A B}}+2 \sum_{\mu \in A} \sum_{\nu \in B} D_{\mu \nu}\langle\nu|\hat{T}| \mu\rangle \\
& +\int\left[\left(V_{N}^{A}+J^{A}+K^{A}\right) \rho^{B}\left(\mathbf{r} \mid \mathbf{r}^{\prime}\right)\right]_{\mathbf{r}^{\prime}=\mathbf{r}} d \mathbf{r} \\
& +\int\left[\left(V_{N}^{B}+J^{B}+K^{B}\right) \rho^{A}\left(\mathbf{r} \mid \mathbf{r}^{\prime}\right)\right]_{\mathbf{r}^{\prime}=\mathbf{r}} d \mathbf{r} .
\end{aligned}
$$

Note that this logic is only applicable when the corresponding potential operator $\hat{Q}$ can be separated into one-atom contributions. Otherwise, the contributions are assigned simply according to the one- or two-center character of the $\langle\nu|\hat{Q}| \mu\rangle$ matrix elements. Thus, the product $D_{\mu \nu}\langle\nu|\hat{Q}| \mu\rangle$ enters the two-atom energy $E_{Q}^{A B}$ if $\mu \epsilon A$ and $\nu \epsilon B$, or the one-atom energy $E_{Q}^{A}$ if the basis functions $\mu$ and $\nu$ belong to the same 
atom $A$. This concept has been applied by Mayer to separate the kinetic-energy contributions, since the kinetic-energy operator $\hat{T}=-(1 / 2) \nabla^{2}$ cannot be ascribed any atomic character in a straightforward manner. The same approach should be valid for any delocalized external potential such as electrostatic field. However, it cannot be applied to the local exchange-correlation term in the DFT due to the fact that the exchange-correlation energy is mainly localized in the areas of electron-density concentration. Numerically, an attempt to use the sum of interatomic terms $D_{\mu \nu}\left\langle\nu\left|\varepsilon_{\mathrm{xc}}\right| \mu\right\rangle$ within the DFT results to underestimated absolute values of the twoatom exchange-correlation energy contributions, which often yields unphysically repulsive two-atom energies for bonded atoms. One example of such inadequate behavior is the positive two-atom interaction energy for $\mathrm{N}_{2}, \mathrm{CO}$, and $\mathrm{F}_{2}$ molecules.

\section{DFT ENERGY DECOMPOSITION}

We are now in a position to derive the decomposition of the DFT energy. Recall that the DFT energy expression is identical to the Hartree-Fock energy expression, except that the Hartree-Fock exchange term [Eq. (1)] is replaced by the exchange-correlation energy,

$$
E_{\mathrm{xc}} \equiv E_{\mathrm{xc}}[\rho]=\int \varepsilon_{\mathrm{xc}}(\mathbf{r}) \rho(\mathbf{r}) d \mathbf{r},
$$

where $E_{\mathrm{xc}}[\rho]$ and $\varepsilon_{\mathrm{xc}}$ are the exchange-correlation functional and the exchange-correlation energy density per electron, ${ }^{12}$ respectively. To divide $E_{\mathrm{xc}}$ into two-atom contributions, we need to represent $\varepsilon_{\mathrm{xc}}$ as a sum of atomic contributions. Due to nonlinear character of $\varepsilon_{\mathrm{xc}}$, we cannot simply assign $\varepsilon_{A}^{\mathrm{xc}}$ $\equiv \varepsilon_{\mathrm{xc}}\left[\rho^{A}\right]$ to the one-atom exchange-correlation contribution of atom $A$, as we did for the Coulomb energy [Eq. (5)]. The solution to the problem is given by a linear expansion of $\varepsilon_{\mathrm{xc}}$ in terms of an atom-centered basis set $\left\{\chi_{\kappa}\right\}$,

$$
\varepsilon_{\mathrm{xc}}(\mathbf{r}) \approx \sum_{\kappa}^{\text {all }} \xi_{\kappa} \chi_{\kappa}(\mathbf{r})=\sum_{A} \sum_{\kappa \in A} \xi_{\kappa} \chi_{\kappa}(\mathbf{r}),
$$

hence the one-atom contribution of atom $A$ is

$$
\varepsilon_{\mathrm{xc}}^{A}(\mathbf{r})=\sum_{\kappa \in A} \xi_{\kappa} \chi_{\kappa}(\mathbf{r})
$$

Note that expansions of such kind are widely used in the computational DFT to facilitate the numerical evaluation of the Coulomb potentials. To minimize the numerical error, an auxiliary basis set, which is typically much larger than the orbital basis set, is usually employed in such cases. However, for the sake of consistency we refrain from using a different basis for expansion (10). As a compromise, for practical calculations, we produced the basis $\left\{\chi_{\kappa}\right\}$ by completely decontracting the basis set $\{|\mu\rangle\}$. The coefficients of expansion (10) can be obtained noniteratively as the solution of the linear system,

$$
\sum_{\kappa}^{\text {all }} S_{\lambda \kappa} \xi_{\kappa}=B_{\lambda}
$$

given by

$$
\xi_{\kappa}=\sum_{\lambda}\left(S^{-1}\right)_{\kappa \lambda} B_{\lambda}
$$

where $B_{\lambda}=\int \varepsilon_{\mathrm{xc}}(\mathbf{r}) \chi_{\lambda}(\mathbf{r}) d \mathbf{r}$. Combining ansatz (8) and the definition (11) yields the following formulas for the two- and one-atom exchange-correlation energies:

$$
\begin{aligned}
& E_{\mathrm{xc}}^{A}=\int \rho^{A}(\mathbf{r}) \varepsilon_{\mathrm{xc}}^{A}(\mathbf{r}) d \mathbf{r}, \\
& E_{\mathrm{xc}}^{A B}=\int \varepsilon_{\mathrm{xc}}^{B}(\mathbf{r}) \rho^{A}(\mathbf{r}) d \mathbf{r}+\int \varepsilon_{\mathrm{xc}}^{A}(\mathbf{r}) \rho^{B}(\mathbf{r}) d \mathbf{r} .
\end{aligned}
$$

Note, however, that there is some arbitrariness in the choice of the original expansion for $\varepsilon_{\mathrm{xc}}(\mathbf{r})$. In principle, in place of $\varepsilon_{\mathrm{xc}}$, any function of the form $w(\mathbf{r}) \varepsilon_{\mathrm{xc}}(\mathbf{r})$ can be employed, where $w(\mathbf{r})$ is an arbitrary positive continuous weighting function of spatial coordinates. Then the above expressions will be modified as follows:

$$
\begin{aligned}
& w(\mathbf{r}) \varepsilon_{\mathrm{xc}}(\mathbf{r}) \approx \sum_{\kappa}^{\text {all }} \xi_{\kappa} \chi_{\kappa}(\mathbf{r}), \\
& B_{\lambda} \equiv \int w(\mathbf{r}) \varepsilon_{\mathrm{xc}}(\mathbf{r}) \chi_{\lambda}(\mathbf{r}), \\
& \varepsilon_{\mathrm{xc}}^{A}(\mathbf{r})=\sum_{\kappa \in A} \frac{\xi_{\kappa} \chi_{\kappa}(\mathbf{r})}{w(\mathbf{r})} .
\end{aligned}
$$

The choice of $w(\mathbf{r})$ will have only a small influence on the magnitude of the total exchange energy $E_{\mathrm{xc}}$, which will vanish in the limit of complete basis set. However, it strongly affects the shape of $\varepsilon_{\mathrm{xc}}^{A}(\mathbf{r})$ and, consequently, the individual contributions $E_{\mathrm{xc}}^{A}$ and $E_{\mathrm{xc}}^{A B}$. The trivial choice of $w(\mathbf{r})=1$ fails, because the fit of $\xi_{\kappa}$ then equally covers the area between the nuclei and the remote area and eventually improves the latter at the expense of the chemically important region between the nuclei. In fact, in the nuclear area substantial oscillations of the total approximate $\varepsilon_{\mathrm{xc}}(\mathbf{r})$ are observed. To increase the quality of the fit in the internuclear region, a positive power of $\rho(\mathbf{r})$ can be used as the weighting function. We preferred to choose the weighting function to be the square root of the density $w(\mathbf{r})=\rho^{1 / 2}(\mathbf{r})$. A similar quantity $(\rho / N)^{1 / 2}$ is sometimes referred to as the "density orbital." 13 By this means, we decrease the importance of the remote areas in favor of nuclear and internuclear regions. This is a special choice of $w(\mathbf{r})$, because it ensures that the approximate exchangecorrelation energy density per electron [Eq. (10)] converges to zero or a nonzero constant at $\mathbf{r} \rightarrow \infty$. On the contrary, powers of $\rho(\mathbf{r})$ higher than $1 / 2$ yield infinitely increasing $\varepsilon_{\mathrm{xc}}(\mathbf{r})$ at $\mathbf{r} \rightarrow \infty$, while lower powers [including $w(\mathbf{r})=1$ ] force an exponential decay of $\varepsilon_{\mathrm{xc}}(\mathbf{r})$. In both alternative cases tested, $w(\mathbf{r})=1$ and $w(\mathbf{r})=\rho(\mathbf{r})$, the individual atomic contributions $\varepsilon_{\mathrm{xc}}^{A}(\mathbf{r})$ exhibit erratic behavior, including changing the sign around the nuclear region. The behavior of 


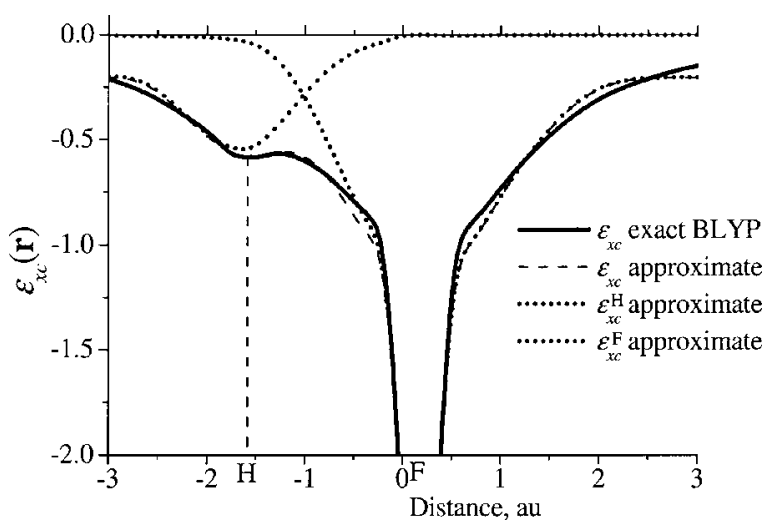

FIG. 1. Exchange-correlation BLYP energy density per electron $\varepsilon_{\mathrm{xc}}(\mathbf{r})$ in the $\mathrm{HF}$ molecule along the line connecting the nuclei in atomic units. The heavy solid line represents the exact BLYP values; the dashed line represents the total approximate values [Eq. (10)]; the dotted lines depict the atomic components of the approximate exchange-correlation energy density per electron $\varepsilon_{\mathrm{xc}}^{\mathrm{H}}(\mathbf{r})$ and $\varepsilon_{\mathrm{xc}}^{\mathrm{F}}(\mathbf{r})$.

the total $\varepsilon_{\mathrm{xc}}(\mathbf{r})$ as well as of the individual contributions $\varepsilon_{\mathrm{xc}}^{\mathrm{H}}(\mathbf{r})$ and $\varepsilon_{\mathrm{xc}}^{\mathrm{F}}(\mathbf{r})$ in the HF molecule calculated using $w(\mathbf{r})$ $=\rho^{1 / 2}(\mathbf{r})$ is illustrated in Fig. 1 for the case of the BLYP functional. It demonstrates that the approximate $\varepsilon_{\mathrm{xc}}(\mathbf{r})$ closely follows the exact BLYP curve. The one-atom contributions $\varepsilon_{\mathrm{xc}}^{\mathrm{H}}(\mathbf{r})$ and $\varepsilon_{\mathrm{xc}}^{\mathrm{F}}(\mathbf{r})$ have pronounced maxima at their respective nuclear positions, and decaying smoothly in the area of the other atom. Thus, a clear spatial separation of $\varepsilon_{\mathrm{xc}}^{A}(\mathbf{r})$ is provided given this choice of $w(\mathbf{r})$.

Expansion (10) is rather accurate but certainly not exact when a finite basis set $\chi_{\kappa}$ is used. The difference between the exact and approximate exchange-correlation energy densities per electron given by

$$
\Delta \varepsilon_{\mathrm{xc}}=\varepsilon_{\mathrm{xc}}(\mathbf{r})-\sum_{\kappa} \frac{\xi_{\kappa} \chi_{\kappa}(\mathbf{r})}{w(\mathbf{r})}=\varepsilon_{\mathrm{xc}}(\mathbf{r})-\sum_{A} \varepsilon_{\mathrm{xc}}^{A}(\mathbf{r})
$$

can be formally considered as a small additional delocalized external potential. The corresponding contribution to the total DFT energy is

$$
\begin{aligned}
\Delta E_{\mathrm{xc}} & =\int \Delta \varepsilon_{\mathrm{xc}} \rho(\mathbf{r}) d \mathbf{r}=\sum_{\mu} \sum_{\nu} D_{\mu \nu}\left\langle\nu\left|\Delta \varepsilon_{\mathrm{xc}}\right| \mu\right\rangle \\
& =\sum_{\mu} \sum_{\nu} D_{\mu \nu}\left\langle\nu\left|\left(\varepsilon_{\mathrm{xc}}(\mathbf{r})-\sum_{\kappa}^{\text {all }} \frac{\xi_{\kappa} \chi^{\kappa}(\mathbf{r})}{w(\mathbf{r})}\right)\right| \mu\right\rangle .
\end{aligned}
$$

Since $\Delta \varepsilon_{\mathrm{xc}}$ is a small and presumably relatively smooth function, the matrix elements $D_{\mu \nu}\left\langle\nu\left|\Delta \varepsilon_{\mathrm{xc}}\right| \mu\right\rangle$ can be readily assigned to one- and two-atom contributions. In view of the small value of the $\Delta \varepsilon_{\mathrm{xc}}$ correction, it has not been implemented in our code. Its main purpose is to make the DFT energy decomposition exact.

We can consider expansion (10) from a slightly different standpoint. Let us make use of the resolution of identity within the subspace spanned by the given basis set in the form

$$
\hat{I} \approx \hat{I}_{b}=\sum_{\lambda \kappa}|\kappa\rangle\left(S^{-1}\right)_{\lambda \kappa}\langle\lambda|
$$

where the summation runs over all the basis functions and $S^{-1}$ is the total inverse overlap matrix. This resolution of identity becomes exact in the limit of the complete basis set. Upon multiplying it by $w(\mathbf{r}) \varepsilon_{\mathrm{xc}}(\mathbf{r})$ and recalling the definitions of $\xi_{\kappa}$ and $B_{\lambda}$ [Eqs. (12) and (14)], we obtain

$$
\begin{aligned}
\hat{I}_{b}\left|w \varepsilon_{\mathrm{xc}}\right\rangle & =\sum_{\lambda \kappa}\left\langle\lambda \mid w \varepsilon_{\mathrm{xc}}\right\rangle\left(S^{-1}\right)_{\lambda \kappa}|\kappa\rangle \\
& =\sum_{\lambda \kappa} B_{\lambda}\left(S^{-1}\right)_{\lambda \kappa}|\kappa\rangle=\sum_{\kappa} \xi_{\kappa}|\kappa\rangle,
\end{aligned}
$$

which demonstrates the equivalence of ansatz (15) and the expansion (10). Note that decomposition (15) is pretty much within the spirit of Mayer's early work, ${ }^{4}$ with the difference that the inverse overlap matrix in projector (15) involves all pairs the basis function, not only those belonging to one or two atoms.

Finally, we summarize the above derivations by giving the working formulas for Mayer's Hartree-Fock energy decomposition $^{5}$ and the present DFT energy decomposition,

$$
\begin{aligned}
E_{A}^{\mathrm{HF}}= & \sum_{\mu \in A} \sum_{\nu \in A} D_{\mu \nu}\langle\nu|\hat{T}| \mu\rangle-\sum_{\mu \in A} \sum_{\nu}^{\text {all }} D_{\mu \nu}\left\langle\nu\left|\frac{Z_{A}}{r_{A}}\right| \mu\right\rangle \\
& +\frac{1}{2} \sum_{\mu \in A} \sum_{\rho \in A} \sum_{\sigma, \nu}^{\text {all }} D_{\mu \nu} D_{\rho \sigma}(\mu \nu \mid \rho \sigma) \\
& -\frac{1}{4} \sum_{\mu \in A} \sum_{\rho \in A} \sum_{\sigma, \nu}^{\text {all }} D_{\mu \sigma} D_{\rho \nu}(\mu \nu \mid \rho \sigma), \\
E_{A B}^{\mathrm{HF}}= & \frac{Z_{A} Z_{B}}{R_{A B}}+2 \sum_{\mu \in A} \sum_{\nu \in B} D_{\mu \nu}\langle\nu|\hat{T}| \mu\rangle \\
& -\sum_{\mu \in A} \sum_{\nu} D_{\mu \nu}\left\langle\nu\left|\frac{Z_{B}}{r_{B}}\right| \mu\right\rangle-\sum_{\mu \in B} \sum_{\nu} D_{\mu \nu}\left\langle\nu\left|\frac{Z_{A}}{r_{A}}\right| \mu\right\rangle \\
& +\sum_{\mu \in A} \sum_{\rho \in B} \sum_{\sigma, \nu}^{\text {all }} D_{\mu \nu} D_{\rho \sigma}(\mu \nu \mid \rho \sigma) \\
& -\frac{1}{2} \sum_{\mu \in A} \sum_{\rho \in B} \sum_{\sigma, \nu}^{\text {all }} D_{\mu \sigma} D_{\rho \nu}(\mu \nu \mid \rho \sigma),
\end{aligned}
$$




$$
\begin{aligned}
E_{A}^{\mathrm{DFT}}= & \sum_{\mu \in A} \sum_{\nu \in A} D_{\mu \nu}\langle\nu|\hat{T}| \mu\rangle-\sum_{\mu \in A} \sum_{\nu}^{\text {all }} D_{\mu \nu}\left\langle\nu\left|\frac{Z_{A}}{r_{A}}\right| \mu\right\rangle \\
& +\frac{1}{2} \sum_{\mu \in A} \sum_{\rho \in A} \sum_{\sigma, \nu}^{\text {all }} D_{\mu \nu} D_{\rho \sigma}(\mu \nu \mid \rho \sigma) \\
& -\int_{\rho^{A}(\mathbf{r}) \varepsilon_{\mathrm{xc}}^{A}(\mathbf{r}) d \mathbf{r},} \\
E_{A B}^{\mathrm{DFT}}= & \frac{Z_{A} Z_{B}}{R_{A B}}+2 \sum_{\mu \in A} \sum_{\nu \in B} D_{\mu \nu}\langle\nu|\hat{T}| \mu\rangle \\
& -\sum_{\mu \in A} \sum_{\nu}^{\mathrm{all}} D_{\mu \nu}\left\langle\nu\left|\frac{Z_{B}}{r_{B}}\right| \mu\right\rangle-\sum_{\mu \in B} \sum_{\nu} D_{\mu \nu}\left\langle\nu\left|\frac{Z_{A}}{r_{A}}\right| \mu\right\rangle \\
& +\sum_{\mu \in A} \sum_{\rho \in B} \sum_{\sigma, \nu}^{\mathrm{all}} D_{\mu \nu} D_{\rho \sigma}(\mu \nu \mid \rho \sigma)-\int \varepsilon_{\mathrm{xc}}^{B}(\mathbf{r}) \rho^{A}(\mathbf{r}) d \mathbf{r} \\
& -\int_{\varepsilon_{\mathrm{xc}}^{A}(\mathbf{r}) \rho^{B}(\mathbf{r}) d \mathbf{r} .}(17)
\end{aligned}
$$

Note that hybrid functionals need a special treatment, in which case the DFT exchange-correlation contribution must be calculated according to formula (17), and the fraction of the Hartree-Fock exchange must be evaluated by (16). It should be also mentioned that expansion (14) becomes illconditioned if the overlap matrix is singular or nearly singular. In such a situation, a slightly modified projector should be used instead of $S^{-1}$. It can be built from the linearly independent functions obtained by means of Löwdin's “canonical" orthogonalization procedure. ${ }^{14}$ Nevertheless, such a situation did not occur in the molecular systems presented in this work.

\section{RESULTS AND DISCUSSION}

Using formulas (17), we calculated one- and two-atom energy components for a series of covalent molecules $\mathrm{C}_{2} \mathrm{H}_{6}$, $\mathrm{C}_{2} \mathrm{H}_{4}, \mathrm{C}_{2} \mathrm{H}_{2}, \mathrm{HF}, \mathrm{HCl}, \mathrm{N}_{2}, \mathrm{~F}_{2}, \mathrm{Cl}_{2}, \mathrm{CO}, \mathrm{H}_{2} \mathrm{CO}, \mathrm{H}_{2} \mathrm{O}$, $\mathrm{CH}_{3} \mathrm{OH}, \mathrm{H}_{2} \mathrm{O}_{2}, \mathrm{CO}_{2}, \mathrm{HCN}, \mathrm{NH}_{3}, \mathrm{~N}_{2} \mathrm{H}_{4}$, and cis- and transdiimide $\mathrm{N}_{2} \mathrm{H}_{2}$, as well as for the water dimer $\left(\mathrm{H}_{2} \mathrm{O}\right)_{2}$ and for the ionic system LiF. The geometry optimization and the evaluation of the molecular orbitals needed for our calculations have been performed using the GAUSSIAN 03 package ${ }^{15}$ with a number of functionals and the $6-31 G^{* *}$ basis set with $5 d$ polarization functions. They include Slater's $(3 / 4)(3 / \pi)^{1 / 3} \rho^{4 / 3}$ local exchange functional, BLYP, ${ }^{16}$ and $\mathrm{PBEPBE}^{17}$ gradient-corrected exchange-correlation functionals as well as the Becke-97 sophisticated hybrid functional. ${ }^{18}$ Thus, a broad range of exchange-correlation functionals is covered. The necessary integrations have been performed using our own code, which implements Becke's partition scheme. ${ }^{19}$ A Lebedev grid for spherical integration with 230 grid points ${ }^{20}$ was borrowed from the DFT repository. ${ }^{21}$ The Chebyshev scheme for radial integration with a total of 135 radial grid points has been used throughout.
For the sake of comparison the Hartree-Fock energy components have also been calculated according to Mayer's formulas (16). The results of the calculations are reported in Table I.

One general observation made from these results is that all the two-atom energies obtained are "on the chemical scale," i.e., they have the order of magnitude typical of dissociation energies of chemical bonds of various kinds. In particular, atoms connected by a strong covalent bond exhibit large negative (attractive) two-atom energies of $100 \mathrm{kcal} \mathrm{mol}^{-1}$ and more, while nonbound atoms have much smaller values, typically within $10 \mathrm{kcal} \mathrm{mol}^{-1}$ and below, which can be either negative or positive (repulsive). In the series of the two-carbon hydrocarbons, the $\mathrm{C}-\mathrm{C}$ diatomic energy strongly increases when going $\mathrm{C}_{2} \mathrm{H}_{6} \rightarrow \mathrm{C}_{2} \mathrm{H}_{4}$ $\rightarrow \mathrm{C}_{2} \mathrm{H}_{2}$, which is consistent both with the known dissociation energies and with the traditional ideas about the chemical bond. The comparison between the methods shows that all the DFT-based approaches yield a much smaller $\mathrm{C}-\mathrm{C}$ two-atom energy in ethane than the Hartree-Fock. For ethylene, the difference between the DFT and Hartree-Fock is much smaller, but it increases again for acetylene. The $\mathrm{C}-\mathrm{H}$ two-atom energies behave more uniformly, having only minor variations between the methods other than the Slater functional. Some difference appears for the C $\cdots H$ nonbonding interactions. The Hartree-Fock two-atom energies are repulsive for ethane and ethylene, but slightly attractive for acetylene. The DFT values are all slightly attractive, with two-atom energies up to $9 \mathrm{kcal} \mathrm{mol}^{-1}$. The Hartree-Fock $\mathrm{H} \cdot \cdot \mathrm{H}$ interaction energies are repulsive or very slightly attractive for ethane and noticeably repulsive for ethylene and acetylene. The DFT $\mathrm{H} \cdot \cdot \mathrm{H}$ interaction energies behave differently for ethane, being slightly negative for all functionals but Becke-97 gives a marginal positive value.

Obviously, any strict comparison of the two-atom energies with dissociation energies or other observables is not particularly helpful, as they have a different meaning. Nonetheless, we can define group interaction energy as the sum of the two-atom interaction energies between two given groups of atoms. If we contrast the experimental bond dissociation energies with the $\mathrm{CH}_{n}-\mathrm{CH}_{n}$ group interaction energies, we see that they reasonably correlate to each other. Though this observation certainly cannot be generalized for other types of bonds, it shows that at least for hydrocarbons the two-atom interaction energy is a clear indicator of the chemical bond strength.

Going further down the Table I, we see that two-atom interaction energies are reasonably similar to the dissociation energies also for the $\mathrm{HF}$ and $\mathrm{HCl}$ molecules. Note that the Hartree-Fock two-atom interaction energies are identical for both molecules, whereas the DFT values correctly reproduce the stronger bond for the HF case. For the $\mathrm{N}_{2}$ and $\mathrm{CO}$ molecules, both at the Hartree-Fock and DFT levels, the twoatom energies are much lower than the extremely high bond dissociation energies. For the dihalogen molecules the situation is opposite: the two-atom energy is higher than the dissociation energy. The DFT values give the correct interpretation of a stronger $\mathrm{Cl}-\mathrm{Cl}$ bond than the $\mathrm{F}-\mathrm{F}$ bond, while the Hartree-Fock results fail to do so. The formaldehyde values 
TABLE I. Two-atom energies for a number of molecules calculated at their respective optimized geometries in $\mathrm{kcal} \mathrm{mol}^{-1}$.

\begin{tabular}{|c|c|c|c|c|c|}
\hline Molecule/bond & $\mathrm{RHF}^{\mathrm{a}}$ & Slater & BLYP & PBEPBE & Becke-97 \\
\hline \multicolumn{6}{|l|}{$\mathrm{C}_{2} \mathrm{H}_{6}$} \\
\hline $\mathrm{C}-\mathrm{C}$ & -120.49 & -63.89 & -85.97 & -91.71 & -79.36 \\
\hline $\mathrm{C}-\mathrm{H}$ & -108.20 & -83.73 & -112.93 & -117.88 & -101.02 \\
\hline $\mathrm{C} \cdots \mathrm{H}$ & 4.38 & -7.22 & -7.28 & -8.64 & -5.07 \\
\hline $\mathrm{H} \cdots \mathrm{H}^{\mathrm{gem}}$ & 8.54 & -0.31 & -4.92 & -2.01 & 0.44 \\
\hline $\mathrm{H} \cdots \mathrm{H}^{\mathrm{vic} \text { gauch }}$ & -0.63 & -0.53 & -1.24 & -0.49 & -0.51 \\
\hline $\mathrm{CH}_{3}-\mathrm{CH}_{3}^{\mathrm{b}}$ & -79.07 & -93.61 & -128.41 & -132.57 & -97.74 \\
\hline \multicolumn{6}{|l|}{$\mathrm{C}_{2} \mathrm{H}_{4}$} \\
\hline $\mathrm{C}-\mathrm{C}$ & -167.76 & -112.00 & -168.79 & -175.72 & -147.47 \\
\hline $\mathrm{C}-\mathrm{H}$ & -111.32 & -78.09 & -110.78 & -110.90 & -94.84 \\
\hline $\mathrm{C}-\mathrm{H}$ & 5.99 & -4.08 & -5.55 & -5.57 & -2.32 \\
\hline $\mathrm{H} \cdots \mathrm{H}^{\mathrm{gem}}$ & 10.98 & 6.99 & 4.91 & 6.88 & 8.28 \\
\hline $\mathrm{H} \cdots \mathrm{H}^{\mathrm{vic}}$ & 3.91 & 4.83 & 3.64 & 4.57 & 4.58 \\
\hline $\mathrm{CH}_{2}-\mathrm{CH}_{2}^{\mathrm{b}}$ & -136.27 & -121.08 & -187.38 & -190.88 & -149.17 \\
\hline \multicolumn{6}{|l|}{$\mathrm{C}_{2} \mathrm{H}_{2}$} \\
\hline $\mathrm{C}-\mathrm{C}$ & -306.61 & -161.55 & -263.80 & -269.17 & -230.06 \\
\hline $\mathrm{C}-\mathrm{H}$ & -97.47 & -76.72 & -113.65 & -108.57 & -89.23 \\
\hline $\mathrm{C} \cdots \mathrm{H}$ & -11.75 & -0.95 & -0.56 & -1.94 & -2.00 \\
\hline $\mathrm{H} \cdots \mathrm{H}$ & 3.46 & 0.96 & 0.04 & 0.95 & 1.49 \\
\hline $\mathrm{CH}-\mathrm{CH}^{\mathrm{b}}$ & -326.65 & -162.49 & -264.89 & -272.09 & -232.56 \\
\hline $\mathrm{HF}$ & -99.50 & -116.76 & -154.27 & -146.28 & -119.29 \\
\hline $\mathrm{HCl}$ & -99.43 & -66.59 & -98.11 & -93.89 & -81.61 \\
\hline $\mathrm{N}_{2}$ & -86.57 & -73.70 & -160.40 & -165.12 & -116.47 \\
\hline $\mathrm{CO}$ & -83.25 & -35.81 & -117.96 & -110.85 & -68.27 \\
\hline $\mathrm{F}_{2}$ & -100.52 & -53.17 & -79.64 & -79.59 & -66.46 \\
\hline $\mathrm{Cl}_{2}$ & -89.73 & -127.94 & -168.15 & -169.08 & -127.97 \\
\hline \multicolumn{6}{|l|}{$\mathrm{H}_{2} \mathrm{CO}$} \\
\hline $\mathrm{C}=\mathrm{O}$ & -161.70 & -80.41 & -152.35 & -150.55 & -122.66 \\
\hline $\mathrm{C}-\mathrm{H}$ & -107.67 & -91.84 & -124.82 & -121.77 & -100.12 \\
\hline $\mathrm{H} \cdots \mathrm{H}$ & 17.16 & 9.33 & 7.03 & 9.08 & 11.23 \\
\hline \multicolumn{6}{|l|}{$\mathrm{H}_{2} \mathrm{O}$} \\
\hline $\mathrm{O}-\mathrm{H}$ & -123.64 & -120.35 & -154.61 & -151.81 & -131.25 \\
\hline $\mathrm{H} \cdots \mathrm{H}$ & 28.78 & 18.61 & 12.64 & 15.65 & 19.21 \\
\hline $\mathrm{H}-\mathrm{OH}^{\mathrm{b}}$ & -94.86 & -101.74 & -141.97 & -136.16 & -112.04 \\
\hline \multicolumn{6}{|l|}{$\mathrm{CH}_{3} \mathrm{OH}$} \\
\hline $\mathrm{C}-\mathrm{O}$ & -142.14 & -77.01 & -103.52 & -109.24 & -95.00 \\
\hline $\mathrm{C}-\mathrm{H}$ & -104.63 & -78.49 & -107.49 & -110.60 & -93.01 \\
\hline $\mathrm{O}-\mathrm{H}$ & -125.62 & -115.5 & -153.20 & -149.74 & -133.18 \\
\hline \multicolumn{6}{|l|}{$\mathrm{H}_{2} \mathrm{O}_{2}$} \\
\hline $\mathrm{O}-\mathrm{O}$ & -124.60 & -79.12 & -95.30 & -103.71 & -89.74 \\
\hline $\mathrm{O}-\mathrm{H}$ & -98.88 & -98.30 & -133.40 & -129.61 & -109.41 \\
\hline $\mathrm{H} \cdots \mathrm{H}$ & 17.83 & 16.01 & 13.82 & 15.08 & 15.90 \\
\hline $\mathrm{OH}-\mathrm{OH}^{\mathrm{b}}$ & -122.23 & -99.49 & -126.34 & -129.75 & -108.3 \\
\hline \multicolumn{6}{|l|}{$\mathrm{CO}_{2}$} \\
\hline $\mathrm{C}=\mathrm{O}$ & -199.42 & -138.36 & -221.39 & -216.91 & -187.89 \\
\hline $\mathrm{O} \cdots \mathrm{O}$ & 33.81 & 7.68 & 10.39 & 7.98 & 13.01 \\
\hline \multicolumn{6}{|l|}{$\mathrm{HCN}$} \\
\hline $\mathrm{H}-\mathrm{C}$ & -98.44 & -67.20 & -106.69 & -91.71 & -71.19 \\
\hline $\mathrm{C}-\mathrm{N}$ & -195.92 & -117.77 & -217.04 & -206.49 & -167.04 \\
\hline $\mathrm{H}-\mathrm{CN}^{\mathrm{b}}$ & -107.30 & -74.42 & -114.68 & -100.30 & -79.79 \\
\hline \multicolumn{6}{|l|}{$\mathrm{NH}_{3}$} \\
\hline $\mathrm{N}-\mathrm{H}$ & -132.23 & -113.85 & -145.19 & -147.58 & -130.71 \\
\hline $\mathrm{H} \cdots \mathrm{H}$ & 19.87 & 11.25 & 5.63 & 9.23 & 12.24 \\
\hline \multicolumn{6}{|l|}{$\mathrm{N}_{2} \mathrm{H}_{4}$} \\
\hline $\mathrm{N}-\mathrm{N}$ & -128.00 & -77.41 & -87.19 & -98.79 & -88.36 \\
\hline $\mathrm{N}-\mathrm{H}$ & -120.37 & -106.09 & -138.54 & -140.88 & -123.91 \\
\hline $\mathrm{H} \cdots \mathrm{H}^{\mathrm{gem}}$ & 21.41 & 11.19 & 5.28 & 8.80 & 12.12 \\
\hline $\mathrm{H} \cdots \mathrm{H}^{\text {vic gauch }}$ & 12.61 & 10.38 & 7.69 & 9.70 & 10.58 \\
\hline $\mathrm{NH}_{2}-\mathrm{NH}_{2}{ }^{\mathrm{b}}$ & -116.60 & -115.29 & -141.71 & -148.11 & -121.60 \\
\hline $\mathrm{N}_{2} \mathrm{H}_{2}$ cis & & & & & \\
\hline
\end{tabular}


TABLE I. (Continued.)

\begin{tabular}{|c|c|c|c|c|c|}
\hline Molecule/bond & $\mathrm{RHF}^{\mathrm{a}}$ & Slater & BLYP & PBEPBE & Becke-97 \\
\hline $\mathrm{N}=\mathrm{N}$ & -167.32 & -107.77 & -162.94 & -169.54 & -146.03 \\
\hline $\mathrm{N}-\mathrm{H}$ & -97.51 & -90.04 & -125.63 & -123.57 & -102.77 \\
\hline $\mathrm{H} \cdots \mathrm{H}$ & 17.26 & 15.12 & 12.53 & 14.49 & 15.33 \\
\hline $\mathrm{NH}-\mathrm{NH}^{\mathrm{b}}$ & -150.54 & -125.29 & -187.43 & -189.81 & -156.64 \\
\hline \multicolumn{6}{|l|}{$\mathrm{N}_{2} \mathrm{H}_{2}$ trans } \\
\hline $\mathrm{N}=\mathrm{N}$ & -175.81 & -133.83 & -183.05 & -191.59 & -166.16 \\
\hline $\mathrm{N}-\mathrm{H}$ & -100.64 & -81.36 & -115.46 & -113.17 & -96.00 \\
\hline $\mathrm{H} \cdots \mathrm{H}$ & 5.59 & 4.05 & 2.95 & 4.06 & 4.69 \\
\hline $\mathrm{NH}-\mathrm{NH}^{\mathrm{b}}$ & -148.12 & -139.68 & -200.98 & -204.03 & -167.73 \\
\hline \multicolumn{6}{|l|}{$\left(\mathrm{H}_{2} \mathrm{O}\right)_{2}$} \\
\hline \multirow[t]{2}{*}{$\mathrm{O}-\mathrm{H}^{\mathrm{e}}$} & -126.55 & $\ldots^{\mathrm{d}}$ & -160.60 & $\ldots^{\mathrm{d}}$ & -136.78 \\
\hline & -124.94 & $\ldots^{\mathrm{d}}$ & -154.25 & $\ldots^{\mathrm{d}}$ & -132.36 \\
\hline $\mathrm{O}-\mathrm{H}^{h^{\mathrm{e}}}$ & -134.22 & $\ldots^{\mathrm{d}}$ & -159.28 & $\ldots^{\mathrm{d}}$ & -134.34 \\
\hline $\mathrm{O} \cdots \mathrm{H}^{h}$ & -54.94 & $\ldots^{d}$ & -49.07 & $\ldots^{\mathrm{d}}$ & -49.35 \\
\hline \multirow{2}{*}{$\mathrm{H}_{2} \mathrm{O}-\mathrm{H}_{2} \mathrm{O}^{\mathrm{b}}$} & -10.71 & $\ldots{ }^{d}$ & -25.45 & $\ldots{ }^{d}$ & -16.11 \\
\hline & -153.62 & -88.61 & -124.50 & -113.94 & -109.51 \\
\hline
\end{tabular}

ariginal Mayer's decomposition [formula (16)]. Small numerical variations compared to the published values (Ref. 5) are due to our use of spherical rather than Cartesian $d$ functions.

${ }^{\mathrm{b}}$ Group interaction energy of the corresponding fragments, see text.

${ }^{\mathrm{c}}$ The superscript $t$ denotes the terminal (non-hydrogen-bonding) hydrogen.

${ }^{\mathrm{d}}$ No adequate minimum structure has been located.

${ }^{\mathrm{e}}$ The superscript $h$ denotes the hydrogen-bonding hydrogen.

are reasonable in principle, indicating a strong $\mathrm{C}=\mathrm{O}$, a weak repulsive $\mathrm{H} \cdots \mathrm{H}$ interaction, and a $\mathrm{C}-\mathrm{H}$ bond very similar to that in ethane. It disconcerts, however, that the $\mathrm{C}=\mathrm{O}$ twoatom energy in $\mathrm{H}_{2} \mathrm{CO}$ is much larger than in $\mathrm{CO}$. The likely physical reason for the underestimated two-atom energy in $\mathrm{CO}$ and $\mathrm{N}_{2}$ is that a too large proportion of the total energy is put into one-atom components due to a very small interatomic separation in these molecules.

The data for the water molecule give a clear picture of strong $\mathrm{O}-\mathrm{H}$ bonds and substantially repulsive $\mathrm{H} \cdots \mathrm{H}$ interactions. The total $\mathrm{HO}-\mathrm{H}$ interaction energy plausibly correlates with the dissociation energy, the Becke-97 and Hartree-Fock values being the closest to the observable. Methanol displays the values very close to those for water for the $\mathrm{O}-\mathrm{H}$ bond, and marginally lower values than two-atom energies in ethane for the $\mathrm{C}-\mathrm{H}$ bonds. For the $\mathrm{C}-\mathrm{O}$ bond, the interaction energies are clearly lower than those in formaldehyde. The difference is more pronounced in the case of the gradientcorrected functionals than for the Hartree-Fock and Becke-97 methods.
The hydrogen peroxide exhibits the $\mathrm{O}-\mathrm{O}$ two-atom energies consistent among the methods. For the $\mathrm{O}-\mathrm{H}$ interaction in $\mathrm{H}_{2} \mathrm{O}_{2}$ all the functionals used yield the two-atom energies about $20 \mathrm{kcal} \mathrm{mol}^{-1}$ weaker than in water. The vicinal $\mathrm{H} \cdots \mathrm{H}$ interaction is repulsive and determined solely by the Coulomb component. The total $\mathrm{HO}-\mathrm{OH}$ group interaction energy is above $100 \mathrm{kcal} \mathrm{mol}^{-1}$ for all functionals, which is in this case more than twice of the experimental dissociation energy.

The carbon dioxide molecule displays the high two-atom energies for the $\mathrm{C}=\mathrm{O}$ bond that are considerably higher than in the formaldehyde. The $\mathrm{O} \cdots \mathrm{O}$ interaction is detected to be slightly repulsive. Interestingly, there is a significant attractive two-atom exchange component (about $-12 \mathrm{kcal} \mathrm{mol}^{-1}$ ), but it is exceeded by the strong Coulomb repulsion.

The results for the $\mathrm{HCN}$ molecule yield a strong $\mathrm{C} \equiv \mathrm{N}$ bond with a two-atom interaction energy of around $200 \mathrm{kcal} \mathrm{mol}^{-1}$ both at the Hartree-Fock and correlated DFT

TABLE II. Various components of two-atom interaction energy in ethylene, in hartree.

\begin{tabular}{|c|c|c|c|c|c|c|}
\hline & $E_{\mathrm{NN}}$ & $E_{\text {kin }}$ & $E_{\mathrm{eN}}$ & $E_{C}$ & $E_{\mathrm{xc}}(\mathrm{DFT})$ & $E_{x}(\mathrm{HF})$ \\
\hline \multicolumn{7}{|l|}{$\mathrm{C}=\mathrm{C}$} \\
\hline RHF & 14.4682 & 0.7802 & -29.9220 & 15.0629 & $\ldots$ & -0.6565 \\
\hline Slater & 14.1542 & 0.7495 & -29.3516 & 14.8026 & -0.5333 & -0.6724 \\
\hline BLYP & 14.2116 & 0.7558 & -29.3214 & 14.6973 & -0.6123 & -0.6758 \\
\hline PBEPBE & 14.2297 & 0.7501 & -29.5078 & 14.8787 & -0.6307 & -0.6680 \\
\hline Becke-97 & 14.2841 & 0.7578 & -29.6081 & 14.9264 & -0.5954 & -0.6654 \\
\hline \multicolumn{7}{|l|}{$\mathrm{C}-\mathrm{H}$} \\
\hline RHF & 2.9496 & 0.3915 & -5.8324 & 2.6616 & $\cdots$ & -0.3477 \\
\hline Slater & 2.8500 & 0.3315 & -5.6407 & 2.5950 & -0.2601 & -0.3254 \\
\hline BLYP & 2.9026 & 0.3487 & -5.8057 & 2.7029 & -0.3251 & -0.3358 \\
\hline PBEPBE & 2.9004 & 0.3515 & -5.7490 & 2.6444 & -0.3241 & -0.3346 \\
\hline Becke-97 & 2.9177 & 0.3626 & -5.7797 & 2.6534 & -0.3051 & -0.3387 \\
\hline
\end{tabular}


level. The $\mathrm{C}-\mathrm{H}$ two-atom energy is systematically somewhat lower than that for ethane and ethylene, which is in accord with the higher acidity of hydrogen cyanide.

The data for the ammonia molecule strongly resemble those for water. The values for the $\mathrm{N}-\mathrm{H}$ bonds are slightly more attractive for the DFT than that in the Hartree-Fock case, and the $\mathrm{H} \cdots \mathrm{H}$ interaction is detected to be repulsive as expected.

In turn, $\mathrm{N}_{2} \mathrm{H}_{4}$ shows similarity to $\mathrm{H}_{2} \mathrm{O}_{2}$, having $\mathrm{N}-\mathrm{N}$ two-atom energies around $100 \mathrm{kcal} \mathrm{mol}^{-1}$, and clearly repulsive $\mathrm{H} \cdots \mathrm{H}$ interactions.

The two endmost molecules given in the Table I-isomers of diimide-are worth comparing to each other and to hydrazine. The $\mathrm{N} \equiv \mathrm{N}$ bonds in cis- and trans-isomers are calculated to be of comparable strengths, though the values for the cis-isomer are $\sim 20 \mathrm{kcal} \mathrm{mol}^{-1}\left(\sim 8 \mathrm{kcal} \mathrm{mol}^{-1}\right.$ at the Hartree-Fock level) lower than those for the transdiimide. For both diimide isomers they are much higher than for hydrazine, consistently with a higher bond order in $\mathrm{N}_{2} \mathrm{H}_{2}$. The $\mathrm{N}-\mathrm{H}$ two-atom energies are somewhat smaller than for hydrazine. The $\mathrm{H} \cdot \cdot \mathrm{H}$ interactions in $\mathrm{N}_{2} \mathrm{H}_{2}$ are repulsive, more so in the cis-diimide in agreement with smaller $\mathrm{H} \cdots \mathrm{H}$ separation.

The water dimer was included as a case study of a comparatively weak noncovalent interaction such as hydrogen bond. The two-atom $\mathrm{O} \cdot \mathrm{H}$ interaction energies for the hydrogen bond are about $50 \mathrm{kcal} \mathrm{mol}^{-1}$ calculated by all the methods available. The interaction energies between two $\mathrm{H}_{2} \mathrm{O}$ fragments are in the range of $10-25 \mathrm{kcal} \mathrm{mol}^{-1}$ depending on the method used. This is of the order of magnitude of, but substantially higher than, the observed dissociation energy. However, the difference will not be as large if the geometry relaxation is taken into account. The covalent $\mathrm{O}-\mathrm{H}^{h}$ two-atom energies (for the hydrogen atoms involved in the hydrogen bond) are calculated even slightly higher than or roughly equal to the values for the terminal (non-hydrogenbonded) $\mathrm{O}-\mathrm{H}^{t}$ bonds. Thus, it apparently fails to reproduce the weakening $\mathrm{O}-\mathrm{H}$ covalent bond due to the interaction with the second oxygen. However, one can obtain the expected chemical picture of the system by taking into account the changes in the one-atom energies (which have not been included into Table I for the sake of brevity). When considering the sum of the one- and two-atom components for the $\mathrm{O}-\mathrm{H}$ bonds, i.e., $E^{\mathrm{O}}+E^{\mathrm{H}}+E^{\mathrm{OH}}$, we can observe that the total $\mathrm{O}-\mathrm{H}^{h}$ moiety is weaker by about $3.4 \mathrm{kcal} \mathrm{mol}^{-1}$ (at the Hartree-Fock level) or $15.5 \mathrm{kcal} \mathrm{mol}^{-1}$ (at the BLYP level) than the $\mathrm{O}-\mathrm{H}^{t}$ one.

Finally, an example of a predominantly ionic systemlithium fluoride-has strongly attractive two-atom interaction energies, similar to those found for strong covalent bonds.

To reveal the role of the various components of the total two-atom energy, we want to take a look at their values summarized in Table II. They show that the two-atom nuclear repulsion and electron repulsion are of very large magnitude, but they are largely counterbalanced by electron-nuclear attraction. It is noteworthy that the kinetic-energy contribution is substantial. The negative exchange or exchangecorrelation contribution is roughly twice of the total effect of the other terms. Generally, the two-atom interaction energy emerges considerably from all five terms, and it will be probably not fruitful to interpret one of them separately. An exception may take place for long-distance interaction between charged atoms, in which case the exchange will be almost vanishing, but significant interaction energy may still appear due to long-distance Coulomb interactions.

It is important to examine to what extent the two-atom energies depend on the basis set used. Obviously, the results must display some basis-set dependence, since the total energy to be decomposed, the electron density, and the KohnSham orbitals all vary with the basis set. An additional source of the basis-set dependence is that the basis set is also used for the exchange-correlation energy decomposition, which can alter the assignment of different proportion of the exchange-correlation energy density per electron to one or another atom. Another very important contribution to these differences is made by geometry relaxation (vide infra). On the other hand, it is highly desirable that the method do not produce unphysical two-atom energy values, or those completely altering the qualitative interpretation of the results. This requirement should hold true at least as long as moderate-quality basis sets are employed. To check this issue, we repeated the calculation of selected molecules from Table I at the BLYP level using a variety of basis sets. Specifically, the STO-3G, 3-21G, 4-31G, 6-31G, 6-311G, 4 $-31 G^{* *}, 6-31 G^{* *}$, and $6-31++G^{* *}$ were studied. The results of these tests are summarized in Table III. They indicate that the two-atom energies for covalent bonds evaluated using different basis sets agree reasonably to each other. Very large deviations are found only for the acetylene and $\mathrm{CO}_{2}$ molecules when using the $3-21 G$ basis set. In all cases the consistent order of bond strength is observed for the $\mathrm{C}_{2} \mathrm{H}_{2}$ $>\mathrm{C}_{2} \mathrm{H}_{4}>\mathrm{C}_{2} \mathrm{H}_{6}$ sequence. Similarly, the $\mathrm{N}=\mathrm{N}$ in diimide bond is calculated stronger than in hydrazine, except for the $6-31++G^{* *}$ basis. The results for the cis-and trans-diimide are close to each other and consistent throughout the basis sets. For the $\mathrm{C}-\mathrm{O}$ bond, $\mathrm{CO}_{2}>\mathrm{H}_{2} \mathrm{CO}>\mathrm{CH}_{3} \mathrm{OH}$ order holds in all cases. The hydrogen bond two-atom energy in the water dimer is calculated to be around $50 \mathrm{kcal} \mathrm{mol}^{-1}$ for all the basis sets except for STO-3G.

For nonbonding interactions, the relative deviations are larger, but nonbonding interactions are still easy to distinguish based either on their positive sign or the small value (typically within $5 \mathrm{kcal} \mathrm{mol}^{-1}$ ) when they are negative.

For practical purposes it may be advantageous to find out whether Mayer's Hartree-Fock energy decomposition can be applied directly to the Kohn-Sham orbitals. In principle, such a procedure is questionable as in this case the total energy is the nonstationary energy expectation value of the single determinant built up from the Kohn-Sham orbitals, and some of the merits of the DFT will be lost. On the other hand, the shape of the occupied Kohn-Sham orbitals is typically not very much different from that of the canonical Hartree-Fock orbitals, such that the resulting Mayer twoatom energies may be still meaningful. Comparison of the Hartree-Fock and DFT two-atom exchange components is given in the two rightmost columns of Table II for the case of ethylene. The variation in the Hartree-Fock exchange values 
TABLE III. Basis-set dependence of the DFT two-atom energies in $\mathrm{kcal} \mathrm{mol}^{-1}$ calculated using the BLYP functional.

\begin{tabular}{|c|c|c|c|c|c|c|c|c|}
\hline Molecule/bond & STO- $3 G$ & $3-21 G$ & $4-31 G$ & $6-31 G$ & $6-311 G$ & $4-31 G^{* *}$ & $6-31 G^{* *}$ & $6-31++G^{* *}$ \\
\hline \multicolumn{9}{|l|}{$\mathrm{C}_{2} \mathrm{H}_{6}$} \\
\hline $\mathrm{C}-\mathrm{C}$ & -107.86 & -164.04 & -119.89 & -100.87 & -92.36 & -111.71 & -85.97 & -101.77 \\
\hline $\mathrm{C}-\mathrm{H}$ & -82.12 & -128.02 & -114.65 & -110.96 & -120.45 & -110.93 & -112.93 & -127.56 \\
\hline $\mathrm{C} \cdots \mathrm{H}$ & -2.28 & -11.31 & -7.71 & -7.99 & -12.05 & -6.84 & -7.28 & -7.57 \\
\hline $\mathrm{H} \cdots \mathrm{H}^{\mathrm{gem}}$ & 0.81 & 7.80 & -0.09 & -0.61 & 3.02 & -3.92 & -4.92 & 0.42 \\
\hline $\mathrm{H} \cdots \mathrm{H}^{\mathrm{vic} \text { gauch }}$ & -0.57 & 1.84 & -0.43 & -0.39 & 0.97 & -1.36 & -1.24 & -0.90 \\
\hline \multicolumn{9}{|l|}{$\mathrm{C}_{2} \mathrm{H}_{4}$} \\
\hline $\mathrm{C}-\mathrm{C}$ & -127.73 & -252.53 & -197.86 & -174.75 & -162.74 & -182.66 & -168.79 & -181.20 \\
\hline $\mathrm{C}-\mathrm{H}$ & -85.78 & -115.60 & -111.04 & -104.99 & -104.15 & -110.42 & -110.78 & -95.28 \\
\hline $\mathrm{C} \cdots \mathrm{H}$ & -1.37 & -5.24 & -4.69 & -4.28 & -9.05 & -5.83 & -5.55 & -2.41 \\
\hline $\mathrm{H} \cdots \mathrm{H}^{\mathrm{gem}}$ & 4.60 & 10.99 & 5.85 & 6.35 & 7.40 & 4.69 & 4.91 & 12.72 \\
\hline $\mathrm{H} \cdots \mathrm{H}^{\mathrm{vic}}$ & 2.58 & 6.17 & 3.81 & 4.08 & 4.99 & 3.41 & 3.64 & 10.33 \\
\hline \multicolumn{9}{|l|}{$\mathrm{C}_{2} \mathrm{H}_{2}$} \\
\hline $\mathrm{C}-\mathrm{C}$ & -153.26 & -468.39 & -354.88 & -309.47 & -306.70 & -252.68 & -263.80 & -243.69 \\
\hline $\mathrm{C}-\mathrm{H}$ & -97.58 & -98.10 & -105.29 & -99.38 & -100.57 & -111.84 & -113.65 & -99.62 \\
\hline $\mathrm{C} \cdot \cdots \mathrm{H}$ & -0.76 & -2.10 & -2.08 & -2.71 & -12.48 & -0.95 & -0.56 & 6.99 \\
\hline $\mathrm{H} \cdots \mathrm{H}$ & 0.52 & 3.66 & 1.59 & 1.80 & 3.32 & 0.04 & 0.04 & 0.78 \\
\hline $\mathrm{HF}$ & -111.67 & -113.28 & -110.73 & -100.81 & -104.74 & -158.46 & -154.27 & -105.91 \\
\hline $\mathrm{HCl}$ & -93.68 & -126.37 & -98.53 & -97.16 & -106.52 & -114.38 & -98.11 & -81.87 \\
\hline $\mathrm{N}_{2}$ & -81.45 & -296.73 & -220.10 & -155.07 & -129.05 & -192.03 & -160.40 & -73.15 \\
\hline $\mathrm{CO}$ & -42.28 & -151.09 & -137.08 & -93.03 & -100.47 & -133.23 & -117.96 & -133.82 \\
\hline $\mathrm{F}_{2}$ & -143.86 & -142.29 & -130.80 & -130.75 & -131.91 & -97.35 & -79.64 & -92.06 \\
\hline $\mathrm{Cl}_{2}$ & -97.20 & -101.84 & -123.46 & -143.47 & -143.78 & -154.38 & -168.15 & -143.03 \\
\hline \multicolumn{9}{|l|}{$\mathrm{H}_{2} \mathrm{CO}$} \\
\hline $\mathrm{C}=\mathrm{O}$ & -155.24 & -205.88 & -197.73 & -164.75 & -148.97 & -169.90 & -152.35 & -172.35 \\
\hline $\mathrm{C}-\mathrm{H}$ & -100.21 & -142.49 & -128.67 & -125.55 & -126.75 & -122.27 & -124.82 & -107.96 \\
\hline $\mathrm{H} \cdots \mathrm{H}$ & 7.25 & 13.09 & 7.50 & 7.33 & 9.11 & 7.50 & 7.03 & 14.11 \\
\hline \multicolumn{9}{|l|}{$\mathrm{H}_{2} \mathrm{O}$} \\
\hline $\mathrm{O}-\mathrm{H}$ & -101.25 & -134.77 & -140.00 & -133.96 & -133.56 & -151.25 & -154.61 & -126.58 \\
\hline $\mathrm{H} \cdots \mathrm{H}$ & 2.16 & 23.63 & 28.78 & 29.64 & 33.88 & 11.64 & 12.64 & 28.50 \\
\hline \multicolumn{9}{|l|}{$\mathrm{CH}_{3} \mathrm{OH}$} \\
\hline $\mathrm{C}-\mathrm{O}$ & -137.77 & -156.76 & -149.77 & -133.21 & -131.36 & -134.33 & -103.52 & -119.78 \\
\hline $\mathrm{C}-\mathrm{H}$ & -83.60 & -128.65 & -111.03 & -106.33 & -109.13 & -107.28 & -107.49 & -121.01 \\
\hline $\mathrm{O}-\mathrm{H}$ & -99.17 & -121.70 & -133.45 & -126.90 & -121.53 & -147.55 & -153.20 & -120.59 \\
\hline \multicolumn{9}{|l|}{$\left(\mathrm{H}_{2} \mathrm{O}\right)_{2}$} \\
\hline \multirow[t]{2}{*}{$\mathrm{O}-\mathrm{H}^{\mathrm{ta}}$} & -118.06 & $-138.21^{\mathrm{b}}$ & -152.80 & -148.18 & -151.68 & $-151.80^{b}$ & $-160.60^{\mathrm{b}}$ & -127.90 \\
\hline & -100.88 & $-133.85^{\mathrm{b}}$ & -139.62 & -135.13 & -139.00 & $-157.70^{b}$ & $-154.25^{\mathrm{b}}$ & -137.17 \\
\hline $\mathrm{O}-\mathrm{H}^{h \mathrm{c}}$ & -75.46 & $-133.87^{\mathrm{b}}$ & -124.84 & -108.72 & -82.31 & $-157.75^{\mathrm{b}}$ & $-159.28^{\mathrm{b}}$ & -107.92 \\
\hline $\mathrm{O} \cdots \mathrm{H}^{h^{\mathrm{c}}}$ & -33.79 & $-46.88^{\mathrm{b}}$ & -55.77 & -52.94 & -48.63 & $-40.28^{\mathrm{b}}$ & $-49.07^{\mathrm{b}}$ & -53.05 \\
\hline $\mathrm{LiF}$ & -119.64 & -126.95 & -132.73 & -124.08 & -118.11 & -139.90 & -124.50 & -120.34 \\
\hline \multicolumn{9}{|l|}{$\mathrm{H}_{2} \mathrm{O}_{2}$} \\
\hline $\mathrm{O}-\mathrm{O}$ & -135.66 & -134.00 & -127.65 & -127.00 & -134.32 & -114.39 & -95.30 & -117.57 \\
\hline $\mathrm{O}-\mathrm{H}$ & -94.22 & -104.00 & -115.08 & -108.20 & -104.71 & -130.63 & -133.40 & -95.15 \\
\hline $\mathrm{H} \cdots \mathrm{H}$ & 1.96 & 15.29 & 18.09 & 18.23 & 17.70 & 13.10 & 13.82 & 15.69 \\
\hline \multicolumn{9}{|l|}{$\mathrm{CO}_{2}$} \\
\hline $\mathrm{C}=\mathrm{O}$ & -183.00 & -336.86 & -295.85 & -225.23 & -199.31 & -262.80 & -221.39 & -257.36 \\
\hline $\mathrm{O} \cdots \mathrm{O}$ & -0.61 & 19.73 & -1.82 & -11.55 & -10.65 & 15.57 & 10.39 & -5.12 \\
\hline \multicolumn{9}{|l|}{$\mathrm{HCN}$} \\
\hline $\mathrm{H}-\mathrm{C}$ & -86.17 & -100.33 & -117.02 & -113.04 & -119.63 & -103.74 & -106.69 & -88.16 \\
\hline $\mathrm{C}-\mathrm{N}$ & -98.55 & -287.91 & -260.41 & -193.38 & -155.75 & -241.62 & -217.04 & -208.50 \\
\hline \multicolumn{9}{|l|}{$\mathrm{NH}_{3}$} \\
\hline $\mathrm{N}-\mathrm{H}$ & -92.20 & -139.98 & -145.77 & -140.69 & -139.84 & -140.20 & -145.19 & -142.25 \\
\hline $\mathrm{H} \cdots \mathrm{H}$ & 4.05 & 16.50 & 18.00 & 18.84 & 20.90 & 4.68 & 5.63 & 24.41 \\
\hline \multicolumn{9}{|l|}{$\mathrm{N}_{2} \mathrm{H}_{4}$} \\
\hline $\mathrm{N}-\mathrm{N}$ & -113.78 & -139.16 & -139.38 & -136.39 & -156.76 & -112.05 & -87.19 & -162.24 \\
\hline $\mathrm{N}-\mathrm{H}$ & -85.37 & -124.15 & -136.97 & -132.17 & -134.34 & -133.67 & -138.54 & -142.43 \\
\hline $\mathrm{H} \cdots \mathrm{H}^{\mathrm{gem}}$ & 4.66 & 18.84 & 18.22 & 18.27 & 20.28 & 5.00 & 5.28 & 20.38 \\
\hline $\mathrm{H} \cdots \mathrm{H}^{\mathrm{vic} \text { gauch }}$ & 3.25 & 10.22 & 11.18 & 11.38 & 12.09 & 7.23 & 7.69 & 13.09 \\
\hline \multicolumn{9}{|l|}{$\mathrm{N}_{2} \mathrm{H}_{2}$ cis } \\
\hline $\mathrm{N}=\mathrm{N}$ & -148.66 & -278.07 & -244.82 & -206.30 & -184.33 & -187.31 & -162.94 & -138.46 \\
\hline
\end{tabular}


TABLE III. (Continued.)

\begin{tabular}{|c|c|c|c|c|c|c|c|c|}
\hline Molecule/bond & STO- $3 G$ & $3-21 G$ & $4-31 G$ & $6-31 G$ & $6-311 G$ & $4-31 G^{* *}$ & $6-31 G^{* *}$ & $6-31++G^{* * *}$ \\
\hline $\mathrm{N}-\mathrm{H}$ & -84.72 & -120.12 & -129.32 & -124.48 & -117.57 & -125.05 & -125.63 & -101.09 \\
\hline $\mathrm{H} \cdots \mathrm{H}$ & 8.73 & 15.41 & 14.90 & 14.85 & 13.03 & 12.20 & 12.53 & 16.71 \\
\hline \multicolumn{9}{|l|}{$\mathrm{N}_{2} \mathrm{H}_{2}$ trans } \\
\hline $\mathrm{N}=\mathrm{N}$ & -144.10 & -287.28 & -252.19 & -213.98 & -194.27 & -209.56 & -183.05 & -148.70 \\
\hline $\mathrm{N}-\mathrm{H}$ & -77.80 & -107.39 & -121.46 & -116.95 & -113.00 & -113.76 & -115.46 & -97.76 \\
\hline $\mathrm{H} \cdots \mathrm{H}$ & 0.16 & 6.10 & 6.14 & 5.91 & 4.57 & 2.61 & 2.95 & 2.91 \\
\hline
\end{tabular}

The superscript $t$ denotes the terminal (non-hydrogen-bonding) hydrogen.

${ }^{\mathrm{b}}$ No adequate minimum structure has been located.

${ }^{\mathrm{c}}$ The superscript $h$ denotes the hydrogen-bonding hydrogen.

TABLE IV. Two-atom Hartree-Fock energies for selected molecules calculated from the Kohn-Sham orbitals by the original Mayer's decomposition [formula (16)] in $\mathrm{kcal} \mathrm{mol}^{-1}$.

\begin{tabular}{|c|c|c|c|c|c|}
\hline Molecule/bond & RHF & Slater & BLYP & PBEPBE & Becke-97 \\
\hline \multicolumn{6}{|l|}{$\mathrm{C}_{2} \mathrm{H}_{6}$} \\
\hline $\mathrm{C}-\mathrm{C}$ & -120.49 & -139.02 & -151.48 & -139.56 & -136.08 \\
\hline $\mathrm{C}-\mathrm{H}$ & -108.20 & -118.84 & -115.31 & -117.24 & -115.24 \\
\hline $\mathrm{C} \cdot \cdots \mathrm{H}$ & 4.38 & 1.09 & 4.01 & 1.99 & 2.57 \\
\hline $\mathrm{H}^{\cdots} \cdot \mathrm{H}^{\mathrm{gem}}$ & 8.54 & 8.39 & 7.88 & 8.84 & 8.76 \\
\hline $\mathrm{H} \cdots \mathrm{H}^{\mathrm{vic} \text { gauch }}$ & -0.63 & -0.99 & -1.59 & -0.87 & -0.87 \\
\hline \multicolumn{6}{|l|}{$\mathrm{C}_{2} \mathrm{H}_{4}$} \\
\hline $\mathrm{C}-\mathrm{C}$ & -167.76 & -199.33 & -208.66 & -199.16 & -191.43 \\
\hline $\mathrm{C}-\mathrm{H}$ & -111.32 & -119.02 & -117.54 & -117.50 & -115.94 \\
\hline $\mathrm{C} \cdots \mathrm{H}$ & 5.99 & 3.98 & 6.30 & 5.00 & 5.09 \\
\hline $\mathrm{H} \cdots \mathrm{H}^{\mathrm{gem}}$ & 10.98 & 12.24 & 12.34 & 12.54 & 12.27 \\
\hline $\mathrm{H} \cdots \mathrm{H}^{\mathrm{vic}}$ & 3.91 & 5.07 & 4.35 & 4.82 & 4.63 \\
\hline \multicolumn{6}{|l|}{$\mathrm{C}_{2} \mathrm{H}_{2}$} \\
\hline $\mathrm{C}-\mathrm{C}$ & -306.61 & -270.47 & -283.18 & -286.97 & -284.49 \\
\hline $\mathrm{C}-\mathrm{H}$ & -97.47 & -116.81 & -118.47 & -114.76 & -110.88 \\
\hline $\mathrm{C} \cdots \mathrm{H}$ & -11.75 & 8.58 & 14.56 & 9.79 & 5.93 \\
\hline $\mathrm{H} \cdots \mathrm{H}$ & 3.46 & 0.69 & -0.20 & 0.70 & 1.29 \\
\hline $\mathrm{HF}$ & -99.50 & -125.51 & -121.92 & -118.96 & -113.64 \\
\hline $\mathrm{HCl}$ & -99.43 & -114.21 & -113.10 & -110.99 & -109.13 \\
\hline $\mathrm{N}_{2}$ & -86.57 & -177.82 & -179.22 & -170.18 & -149.35 \\
\hline $\mathrm{CO}$ & -83.25 & -142.77 & -142.33 & -134.27 & -121.08 \\
\hline $\mathrm{F}_{2}$ & -100.52 & -128.19 & -124.76 & -123.34 & -118.20 \\
\hline $\mathrm{Cl}_{2}$ & -89.73 & -120.08 & -118.59 & -115.49 & -110.13 \\
\hline \multicolumn{6}{|l|}{$\mathrm{H}_{2} \mathrm{CO}$} \\
\hline $\mathrm{C}=\mathrm{O}$ & -161.70 & -190.49 & -197.03 & -186.83 & -180.14 \\
\hline $\mathrm{C}-\mathrm{H}$ & -107.67 & -127.79 & -126.78 & -123.91 & -119.24 \\
\hline $\mathrm{H} \cdots \mathrm{H}$ & 17.16 & 16.56 & 16.80 & 17.12 & 17.26 \\
\hline \multicolumn{6}{|l|}{$\mathrm{H}_{2} \mathrm{O}$} \\
\hline $\mathrm{O}-\mathrm{H}$ & -123.64 & -137.06 & -131.51 & -130.72 & -129.03 \\
\hline $\mathrm{H} \cdots \mathrm{H}$ & 28.78 & 25.90 & 23.45 & 24.68 & 25.79 \\
\hline \multicolumn{6}{|l|}{$\mathrm{CH}_{3} \mathrm{OH}$} \\
\hline $\mathrm{C}-\mathrm{O}$ & -142.14 & -147.41 & -156.00 & -145.42 & -143.50 \\
\hline $\mathrm{C}-\mathrm{H}$ & -104.63 & -117.70 & -115.70 & -115.48 & -112.72 \\
\hline $\mathrm{O}-\mathrm{H}$ & -125.62 & -129.41 & -127.03 & -125.70 & -125.62 \\
\hline \multicolumn{6}{|l|}{$\left(\mathrm{H}_{2} \mathrm{O}\right)_{2}$} \\
\hline \multirow[t]{2}{*}{$\mathrm{O}-\mathrm{H}^{t^{\mathrm{a}}}$} & -126.55 & $\ldots^{\mathrm{b}}$ & -134.32 & $\ldots{ }^{b}$ & -132.02 \\
\hline & -124.94 & $\ldots{ }^{b}$ & -129.72 & $\ldots{ }^{b}$ & -128.28 \\
\hline $\mathrm{O}-\mathrm{H}^{h^{\mathrm{c}}}$ & -134.22 & $\ldots{ }^{b}$ & -143.06 & $\ldots^{\mathrm{b}}$ & -139.95 \\
\hline $\mathrm{O} \cdots \mathrm{H}^{h}$ & -54.94 & $\ldots$ b & -53.30 & $\ldots \mathrm{b}$ & -55.88 \\
\hline $\mathrm{LiF}$ & -153.62 & -179.3 & -169.10 & -166.87 & -161.35 \\
\hline
\end{tabular}

${ }^{\mathrm{a}}$ The superscript $t$ denotes the terminal (non-hydrogen-bonding) hydrogen.

${ }^{\mathrm{b}}$ No adequate minimum structure has been located.

${ }^{\mathrm{c}}$ The superscript $h$ denotes the hydrogen bonding hydrogen. 
between the functionals is marginal, which shows that the effect of the molecular geometry and the exchangecorrelation functional is small. This holds true even for the Slater functional. The DFT exchange-correlation values $E_{\mathrm{xc}}(\mathrm{DFT})$ vary a little stronger, but agree still very well with the Hartree-Fock results. To examine this issue for a broad number of molecules, the Table IV summarizes the two-atom energies calculated according to formula (16) from the Kohn-Sham orbitals. Note that the difference between the various functionals is here only due to the difference in the orbital shape and molecular geometry, as the same HartreeFock energy expression applies. The important trend recognizable from these results is that the two-atom interaction energies for strong covalent bonds are rather close to the Hartree-Fock values (Table I). The exception is the "notorious" molecules $\mathrm{CO}$ and $\mathrm{N}_{2}$, for which cases the energies obtained using the Kohn-Sham orbitals are nearly twice of the Hartree-Fock values. The situation is slightly different for the unspecific nonbonding interactions. In most such cases, the two-atom energies calculated using the KohnSham orbitals have the same sign as the Hartree-Fock values, i.e., are positive (repulsive), but in some other cases the DFT energies are slightly negative (attractive). Therefore, the positive Hartree-Fock values obtained using the Kohn-Sham orbitals can be helpful to distinguish weak repulsive interactions.

Various effects of the change in geometry, orbitals, and the partitioning scheme on the two-atom energy in the dinitrogen molecule are illustrated on Scheme 1. Let us start with the $\mathrm{HF} / \mathrm{HF}$ value, which corresponds to the Hartree-Fock result already shown in Table I. When moving right on the chart, the MOs are allowed to relax to the Kohn-Sham ones. The Kohn-Sham (KS)//Hartree-Fock (HF) values indicate that the effect of the change in the orbitals is to increase the two-atom energy by 20-30 $\mathrm{kcal} \mathrm{mol}^{-1}$, depending upon the

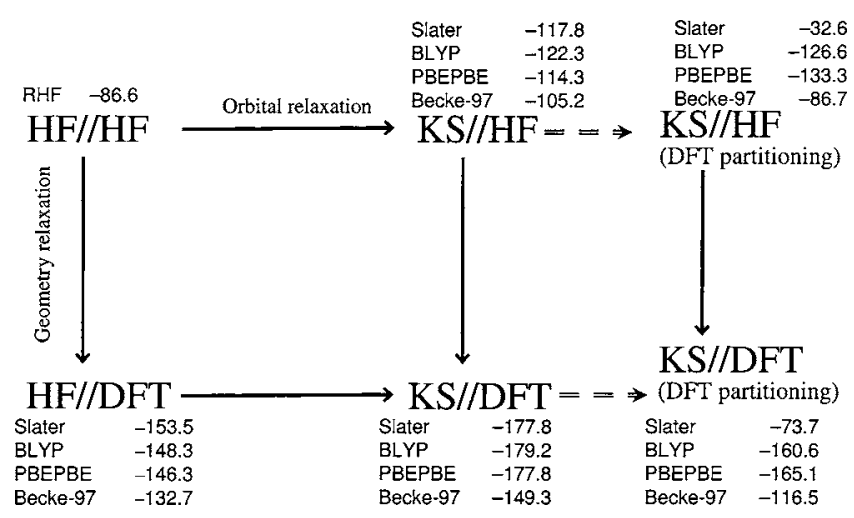

SCHEME 1. Two-atom interaction energy in $\mathrm{N}_{2}$ in $\mathrm{kcal} \mathrm{mol}^{-1}$ calculated at different geometries and density functionals. The notation $X / / Y$ corresponds to the result obtained using orbitals at level $X$ evaluated at the geometry optimized at level $Y$. Mayer's Hartree-Fock partitioning scheme [Eq. (16)] is employed unless otherwise specified.

method. In the next step, the molecular geometry is allowed to relax to the optimized value for the respective functional (KS//DFT). It can be seen that the two-atom energy is further increased by $45-63 \mathrm{kcal} \mathrm{mol}^{-1}$. Thus, the geometry relaxation effect is more important in this case than the orbital relaxation, and hence responsible for most of the differences between the Hartree-Fock and DFT results given in Table I. Finally, the effect of the partitioning scheme employed can be assessed by inspection of the KS//DFT and KS//HF results obtained within the present DFT partitioning, which are shown in the rightmost part of Scheme 1. All the differences between the Hartree-Fock and DFT partitionings are solely due to the different treatment of the exchange-correlation term. In this case the different functionals exhibit larger differences between them. In the case of the Slater exchange, the two-atom energy contribution drops by up to $100 \mathrm{kcal} \mathrm{mol}^{-1}$, and thus the final value is close to the pure Hartree-Fock one. The differences on the DFT exchangecorrelation term for Becke-97 functional are of the same order as the effects of the orbital and geometry relaxations, and thus the DFT results are closer to the Hartree-Fock ones. The remaining functionals behave more uniformly. Introducing the DFT exchange has a smaller influence on the two-atom energy than the geometry and orbital relaxations. The twoatom energy can increase or decrease depending on the geometry considered. In the case of the fully relaxed calculation (KS//DFT), the energy decreases by $10-20 \mathrm{kcal} \mathrm{mol}^{-1}$ to the final values gathered in Table I. This analysis indicates that the main reason for the large differences between the Hartree-Fock and DFT two-atom energies for $\mathrm{N}_{2}$ is the geometry change. The differences due to the use of the KohnSham orbitals can also be important. The effect of the DFT exchange-correlation contribution is relatively small, except for the Slater functional.

\section{CONCLUSIONS}

The present work provides a generalization of Mayer's Hartree-Fock energy decomposition into one- and two-atom components for the DFT case. First, we gave an analysis of Mayer's original decomposition and demonstrated that most terms in it can be represented as an action of an effective one-atom potential operator on a one-atom effective density matrix. The formulation of the DFT energy partitioning given here involves a replacement of the exchange term in Mayer's expression.

Both Hartree-Fock and DFT two-atom energies for strong covalent bonds are within the range of typical bond dissociation energies, i.e., dozens or hundreds of $\mathrm{kcal} \mathrm{mol}^{-1}$. The DFT two-atom interaction energies agree to a reasonable extent with the respective Hartree-Fock values. Typically, both tested gradient-corrected functionals, BLYP and PBEPBE, yield very similar two-atom interaction energies. The hybrid Becke-97 functional usually produces results intermediate between those of Hartree-Fock and BLYP methods. In general, the two-atom energies calculated by different functionals give the results that are roughly as much different as between the DFT and Hartree-Fock.

For nonspecific nonbonding interactions, all the twoatom energies are low in values, mostly within $10 \mathrm{kcal} \mathrm{mol}^{-1}$. They can be either attractive or repulsive, but the DFT results have a slight trend toward small attractive values compared to the Hartree-Fock. The studied case of 
the hydrogen bond, the water dimer, indicates that the hydrogen bond two-atom energies lie between the strong covalent and nonbonding interactions.

The tests performed indicate that the two-atom energies depend quite little upon the basis set, and all the qualitative trends hold true when using moderately sized basis sets.

Technically, Mayer's Hartree-Fock energy analysis can be also applied to the Kohn-Sham orbitals obtained from a DFT calculation. Though this approach is not very founded theoretically, the numerical results are reasonably close to those obtained using canonical Hartree-Fock orbitals. The two-atom energies calculated in this manner for nonbonding interactions can be helpful to distinguish nonspecific repulsive interactions.

In summary, both Mayer's Hartree-Fock and the present DFT energy decomposition present a convenient computational tool for a posteriori interpretation of the bond strength in polyatomic molecules. It has perspectives for studying specific intramolecular interactions such as intramolecular hydrogen bond or agostic interactions.

\footnotetext{
${ }^{1}$ R. F. W. Bader, Atoms in Molecules-A Quantum Theory (Oxford University Press, Oxford, 1990).

${ }^{2}$ A. E. Reed and F. Weinhold, J. Chem. Phys. 78, 4066 (1983); A. E.
}

Reed, R. B. Weinstock, and F. Weinhold, ibid. 83, 735 (1985); A. E. Reed, L. A. Curtiss, and F. Weinhold, Chem. Rev. 88, 899 (1988).

${ }^{3}$ H. Fischer and H. Kollmar, Theor. Chim. Acta 16, 163 (1970).

${ }^{4}$ I. Mayer, Chem. Phys. Lett. 332, 381 (2000).

${ }^{5}$ I. Mayer, Chem. Phys. Lett. 382, 265 (2003).

${ }^{6}$ P. Salvador, M. Duran, and I. Mayer, J. Chem. Phys. 115, 1153 (2001).

${ }^{7}$ I. Mayer and A. Hamza, Theor. Chem. Acc. 105, 360 (2001).

${ }^{8}$ D. R. Alcoba, A. Torre, L. Lain, and R. C. Bochicchio, J. Chem. Phys. 122, 074102 (2005).

${ }^{9}$ P. Salvador and I. Mayer, J. Chem. Phys. 120, 5046 (2004).

${ }^{10}$ A. Sierraalta and G. Frenking, Theor. Chim. Acta 95, 1 (1997).

${ }^{11}$ A. Hamza and I. Mayer, Theor. Chim. Acta 109, 91 (2003).

${ }^{12}$ R. Parr and W. Yang, Density Functional Theory of Atoms and Molecules (Oxford University Press, New York, 1989).

${ }^{13}$ P. R. T. Schipper, O. V. Gritsenko, and E. J. Baerends, Phys. Rev. A 57, 1729 (1998)

${ }^{14}$ P.-O. Löwdin, Adv. Phys. 5, 1 (1956).

${ }^{15}$ M. J. Frisch, G. W. Trucks, H. B. Schlegel et al., Gaussian 03, Revision B.01, Gaussian, Inc., Pittsburgh, PA, 2003.

${ }^{16}$ A. D. Becke, Phys. Rev. A 38, 3098 (1988); C. Lee, W. Yang, and R. G. Parr, Phys. Rev. B 37, 785 (1988); B. Miehlich, A. Savin, H. Stoll, and H. Preuss, Chem. Phys. Lett. 157, 200 (1989).

${ }^{17}$ J. P. Perdew, K. Burke, and M. Ernzerhof, Phys. Rev. Lett. 77, 3865 (1996); 78, 1396 (1997).

${ }^{18}$ A. D. Becke, J. Chem. Phys. 107, 8554 (1997).

${ }^{19}$ A. D. Becke, J. Chem. Phys. 88, 2547 (1988).

${ }^{20}$ V. I. Lebedev, Sib. Math. J. 18, 99 (1977).

${ }^{21}$ Density Functional Repository, Quantum Chemistry Group, CCLRC Daresbury Laboratory, Daresbury, Cheshire, WA4 4AD United Kingdom. 